and on the global Bayley mental ability scores. (Ross G, Boatright S, Auld PAM, Nass R. Specific cognitive abilities in 2-year-old children with subependymal and mild intraventricular hemorrhage. Brain Cogn Oct 1996;32:1-13). (Reprints: Dr Gail Ross, Perinatology Center, New York Hospital, 525 East 68 th Street, New York, NY 10021).

COMMENT. Prior testing of these prematures at age 10 months had shown that S/IVH affected global mental ability and habituation to visual patterns, and prematurity was associated with poorer memory for location. When reevaluated at 2 years, the premature groups with or without hemorrhage did not differ on the visual attention task, but prematures with S/IVH did poorly on the memory for location task and ability to change response set. Memory for location is a function of the caudate nucleus and thalamus and frontal cortex, areas affected by subependymal and intraventricular hemorrhage of prematurity. Fronto-striatal structural changes in the MRI have been demonstrated in patients with ADHD. (Denckla MB. In: Progress in Pediatric Neurology II, Chicago, PNB Publ, 1994:173-176). At a later age, the incidence of ADHD in the S/IVH affected children will be of interest.

Indomethacin prophylaxis against IVH in very low birth weight infants did not result in adverse cognitive or motor outcomes at 36 months, in a study at Yale University School of Medicine. (Ment LR et al. Pediatrics Oct 1996;98:714-718). The authors suggest that the early administration of intravenous low-dose indomethacin to neonates weighing $1250 \mathrm{~g}$ or less is beneficial and does not cause neurodevelopmental delay. However, Dr Henrietta Bada, University of Tennessee, Memphis, advises caution, and slow infusion, because of reported acute cerebral effects. (Commentary. Routine indomethacin prophylaxis: has the time come? Pediatrics Oct 1996;98:784-785).

\title{
EARLY DETECTION OF CEREBRAL PALSY
}

An Early Motor Pattern Profile (EMPP), consisting of 15 tests of muscle tone, reflexes, and movement, organized in a standardized format, was used to identify children with cerebral palsy (CP) in the first year of life at the University of Illinois at Chicago, College of Medicine at Peoria. The items evaluated included head lag, hip abduction, tonic neck reflex, fisting, scissoring, and toe-walking, and a three-point scoring system was applied to each item. In 1247 high-risk infants from a neonatal intensive care unit followed for 36 months, the EMPP identified children at risk for CP by 6 to 12 months of age. The optimal cutoff score of points at 6 months was 10 , and at 12 months it was 4 . The predictive value was approximately 90\%. (Morgan AM, Aldag JC. Early identification of cerebral palsy using a profile of abnormal motor patterns. Pediatrics Oct 1996;98:692-697). (Reprints: Dr Andrew M Morgan, Department of Pediatrics, St Francis Medical Center, 530 NE Glen Oah Ave, Peoria, IL 61637).

COMMENT. The authors emphasize that the EMPP is not a method of diagnosis, but only a step toward more formal evaluation by a pediatric neurologist. False positive and negative results are always a problem with screening procedures of this type, and their sensitivity is dependent on the skill and expertise of the examiner.

Progressive deterioration of dyskinetic cerebral palsy in adult life is reported in the majority of 20 patients examined between 20 and 40 years of age at the Institute of Neurology, London. (Fletcher NA, Marsden CD. Dyskinetic cerebral palsy: a clinical and genetic study. Dev Med Child Neurol 
Oct 1996;38:873-880). Genetic heterogeneity with autosomal recessive and dominant variants was suggested by familial cases, casting doubt on the perinatal hypoxic-ischemic etiology of this form of CP.

\section{SEIZURE DISORDERS}

\section{HERPESVIRUS 6 INFECTION AND FEBRILE SEIZURES}

The link between human herpesvirus-6 (HHV-6) and other viruses and febrile convulsions (FC) in 65 children (mean age 18 months) with a first episode of simple FC (Group 1) compared to 24 children (mean age 19 months) with a febrile syndrome without FC (Group 2), was examined at the University of Modena, and the Civil Hospital of Sassuolo, Italy. HHV-6 was found in 23/65 of group 1 patients and 12/24 of group 2; adenoviruses in 9/65 of group 1 and in $0 / 24$ of group 2 . Of $35 \%$ FC cases testing positive for HHV-6, only $17 \%$ had the typical exanthema. In the HHV-6 infected group, children who developed FC had lower total immunoglobulins, especially IgM. Children with FC were more likely to have a family history of FC and circulating granulocytes. Of 57 patients followed for 2 years, 9 (15\%) had a second FC, and HHV-6 reactivations were three times more frequent in this group. (Bertolani MF, Portolani M, Marotti $\mathrm{F}$ et al. A study of childhood febrile convulsions with particular reference to HHV-6 infection: pathogenic considerations. Child's Nerv Syst Sept 1996;12:534-539). (Respond: Dr Maria F Bertolani, Section of Pediatrics, University of Modena, Largo del Pozzo, 71, I-41100 Modena, Italy).

COMMENT. The authors speculate that several viruses, especially HHV-6, may be implicated in causation of febrile convulsions in two thirds of cases, and may be reactivated to induce recurrences. The heredity factor is also important, involving a reduced immune response to viral infection in susceptible children. Those who develop FC with HHV-6 infection have a marked granulocytosis and reduced immunoglobulins, IgA and IgM. The influence of enhanced cytokine production in FC is unproven.

Febrile seizures caused by fever induced by HHV-6 infection and roseola are not always simple in type. They are frequently prolonged, recurrent, and complex, and sometimes a manifestation of encephalitis or encephalopathy. For additional reports of HHV-6 infection and febrile seizures, see Ped Neur Briefs Sept 1994, and Progress in Pediatric Neurology II, 1994:410-411.

Iron deficiency anemia and febrile convulsions are linked in a study from the University of Naples, Italy. (Pisacane A, Sansone R, Impagliazzo $\mathrm{N}$ et al. BMI 10 Aug 1996;313:343). Anemia (Hgb $<105 \mathrm{~g} / 1$, serum iron $<5.4$ $\mathrm{mcmol} / 1$ ) occurred in $30 \%$ of FC cases compared to $10 \%$ in the non-FC control population. Iron deficiency anemia has also been associated with a case of reversible focal neurologic deficits, and with breath-holding spells. (Progress in Pediatric Neurology I, 1991:397-398).

\section{EARLY TREATMENT OF SEIZURE PREVENTS RECURRENCE}

The rate of occurrence of a second seizure after a single unprovoked generalized tonic-clonic seizure was compared in 45 patients who received immediate anticonvulsant therapy and 42 untreated patients followed for 36 months at the Edith Wolfson Medical Center, Holon, and the Sackler Faculty of Medicine, Tel Aviv, Israel. A second epileptic attack occurred in 29 (71\%) of the untreated group and in $10(22 \%)$ of the treated group. The risk rates for 